\title{
La estructura administrativa para la promoción y defensa de la competencia en Andalucía
}

\author{
Carlos Padrós Reig \\ Profesor Titular de Derecho administrativo. \\ Miembro fundador del grupo de investigación REDERCO \\ (Regulación, Derecho y Competencia). \\ Universitat Autònoma de Barcelona
}

\begin{abstract}
SUMARIO: 1. INTRODUCGIÓN. 2. LA DESCENTRALIZACIÓN DE LA POLÍTICA DE DEFENSA DE LA COMPETENCIA.2.1. Modelo descentralizado versus modelo participativo. 2.2. Efectos de la descentralización sobre el conjunto de la defensa de la competencia. 3. LA LEY DEL PARLAMENTO DE ANDALUCÍA 6/2007, DE 26 DE JUNIO, DE PROMOCIÓN Y DEFENSA DE LA COMPETENCIA DE ANDALUCÍA. 4. SIMILITUDES Y DIFERENCIAS ENTRE LA LEY ESTATAL 15/2007, DE 3 DE JULIO, DE DEFENSA DE LA COMPETENCIA Y LA LEY ANDALUZA 6/2007, DE 26 DE JUNIO. 4.1. Autoridad única: Comisión Nacional de Competencia y Agencia de Defensa de la Competencia de Andalucía. 4.2. Naturaleza jurídica. 4.3. Responsabilidad y rendición de cuentas. 4.4. Miembros, personal y régimen económico. 4.5. Promoción de la competencia (competition advocacy). 5. CONCLUSIONES. 6. BIBLIOGRAFÍA
\end{abstract}

\section{INTRODUCCIÓN}

España experimenta desde 1999 un proceso de descentralización de la política de defensa de la competencia. La defensa de la competencia constituye una política administrativa que tiene por misión velar por el correcto funcionamiento de los mercados y ofrecer protección a los consumidores contra prácticas restrictivas de los operadores. ${ }^{1} \mathrm{Al}$ ser una política administrativa, de-

\footnotetext{
${ }^{1}$ El Preámbulo de la Ley andaluza 6/2007, de 26 de junio, establece que: "La fundamentación económica de estos principios jurídicos se encuentra en que la competencia es un elemento básico para el desarrollo económico y social de cualquier sociedad avanzada. En condiciones de competencia efectiva se asignan los recursos productivos a favor de los operadores o las técnicas
} 
bería adaptarse a la estructura administrativa general que presenta el Estado autonómico.

Este proceso de descentralización se enmarca también en la reforma de la legislación comunitaria emprendida por el Reglamento CE no $1 / 2003$, de 16 de diciembre de 2002, relativo a la aplicación de las normas sobre competencia previstas en los artículos 81 y 82 del Tratado de la Comunidad Europea. Este Reglamento CE propone un sistema administrativo descentralizado para la aplicación de la defensa de la competencia en la Comunidad europea, donde la autoridad comunitaria y las nacionales trabajan dentro de una Red europea de autoridades de defensa de la competencia. En lo institucional, el mismo Tribunal constitucional reconoce que "no sólo es útil sino también obligado para su correcta aplicación el prestar atención al modo en que una determinada institución jurídica ha sido configurada por la normativa comunitaria” (FJ 4 STC 208/99). Así pues, la descentralización española está perfectamente en línea con la comunitaria.

En este contexto, varias Comunidades Autónomas han emprendido la regulación de sus órganos de defensa de la competencia ${ }^{2}$, siendo la Ley del Parlamento de Andalucía 6/2007, de 26 de junio, la última manifestación al respecto.

Estas breves páginas tienen como objetivo analizar la configuración de la estructura institucional de la política de defensa de la competencia en Andalucía, con la novedosa aparición de la Agencia de Defensa de la Competencia de Andalucía (ADCA). Como sea que la Ley 15/2007, de 3 de julio, modifica el marco institucional del Estado, extinguiéndose el Tribunal y el Servicio de Defensa de la Competencia y creándose una Administración independiente como es la Comisión Nacional de Competencia, conviene también efectuar un análisis comparativo de las semejanzas y discrepancias entre el modelo andaluz y el modelo estatal.

más eficientes y, por tanto, se incentiva la innovación y se fomenta la productividad. Esta mejora de la eficiencia productiva se traslada a los consumidores mediante una reducción de los precios o un aumento de la variedad y calidad de los productos ofertados, con la consiguiente mejora del bienestar del conjunto de la sociedad."

${ }^{2}$ Cataluña: Decreto 222/2002 de 17 de agosto. Galicia: Ley 6/2004, de 12 de julio. Madrid: Ley 6/2004 de 28 de diciembre. País Vasco: Decreto 81/2005, de 12 de abril. Extremadura: Ley 2/2005, de 24 de junio. Aragón: Decreto 29/2006, de 24 de enero. Castilla León: Decreto $36 / 2006$, de 25 de mayo. 
Si bien las Comunidades gozan del poder de autoorganizar las estructuras administrativas que deben desarrollar sus competencias, podría ser que la configuración misma de la función a ejecutar impusiera ciertos límites a la libertad autonómica.

\section{LA DESCENTRALIZACIÓN DE LA POLÍTICA DE DEFENSA DE LA COMPETENCIA}

La descentralización de la defensa de la competencia en España fue en cierta medida impuesta por la STC 208/1999, de 11 de noviembre. Este nacimiento por resolución constitucional ha condicionado el modelo administrativo descentralizado actual. La sentencia resuelve sendos recursos de inconstitucionalidad interpuestos por el Gobierno Vasco y por la Generalitat de Cataluña contra la Ley 16/1989. De la sentencia destacan, en primer lugar lo dilatado del plazo de resolución (10 años $)^{3}$, y en segundo lugar que se trata en el fondo de un conflicto de competencias, puesto que lo que se discute es la atribución en exclusiva para todo o parte del mercado español de la política de defensa de la competencia. Según el TC al tratarse de una competencia ejecutiva encuadrable dentro del título de comercio interior, su desarrollo corresponde a la Comunidad. Este desarrollo, sin embargo, tiene algunos límites:

"La competencia ejecutiva que, en materia de defensa de la competencia, cabe atribuir a las Comunidades Autónomas, en virtud de la asunción de comercio interior se halla, pues, limitada a aquellas actuaciones ejecutivas que hayan de realizarse en su territorio y que no afecten al mercado supracomunitario. De modo que no sólo la normación, sino todas las actividades ejecutivas que determinen la configuración real del mercado con trascendencia extracomunitaria habrán de atribuirse al Estado, al que corresponderán, por lo tanto, las actuaciones ejecutivas en relación con aquellas prácticas que puedan alterar la libre competencia en un ámbito supracomunitario o en el conjunto del mercado nacional, aunque tales actuaciones se realicen en el territorio de cualquiera de las Comunidades Autónomas recurrentes; pero, con esas limitaciones, la competencia ejecutiva atribuida a las Comunidades Autónomas por sus respectivos Estatutos, no puede resultar enervada por la legislación del Estado." (FJ 6)

Por tanto, la sentencia abre las puertas a la actividad autonómica en materia de control y defensa de la competencia. Corresponde al Estado enjuiciar aquellas conductas cuya afectación a la libre competencia alcance a más de

\footnotetext{
${ }^{3}$ fecha de interposición de los recursos: 17 de octubre de 1989, fecha de publicación de la sentencia 11 de noviembre de 1999.
} 
una Comunidad o al conjunto del mercado nacional, así como establecer normativamente los puntos de conexión, cosa que se hace en la Ley 1/2002, de 21 de febrero, de coordinación de las competencias del Estado y las Comunidades Autónomas en materia de defensa de la competencia. De hecho, para evitar un vacío normativo, los efectos de la declaración de inconstitucionalidad se demoran hasta la aprobación de la citada Ley.

No existe duda de que lograr el compromiso efectivo de las autoridades autonómicas de defensa de la competencia puede redundar en una mayor efectividad del cumplimiento de las normas de defensa de la competencia. Como ha explicado CASES PALLARÉS, "En España está en curso un proceso de implantación de autoridades autonómicas de protección de la competencia cuyo origen es la Sentencia del Tribunal Constitucional de 11 de noviembre de 1999 y se articula en base a la Ley $1 / 2002$. (...) Este nuevo sistema institucional, que requiere, por tanto, un compromiso cierto de las autoridades autonómicas en la protección de la competencia, puede constituir un impulso decisivo para la aplicación más efectiva de las normas de competencia. Es evidente que ello requiere especialmente mecanismos de coordinación y cooperación entre autoridades, que se están articulando en términos positivos". ${ }^{4}$

Aunque no corresponde a estas páginas efectuar un análisis de la Ley 1/2002 sí podemos decir que se utiliza un enfoque basado en la teoría de los efectos económicos de las conductas. Cuando los efectos se limiten al ámbito territorial autonómico será competente el organismo ad hoc creado. Por el contrario, cuando la alteración de la competencia alcance el ámbito supra autonómico, tendrá competencia el Estado. Esa alteración puede producirse por afectar a la unidad de mercado o por producirse una ruptura del equilibrio económico adecuado y justo entre las distintas partes del territorio español o por obstaculizar la libre circulación en todo el territorio nacional.

Como indican NADAL y ROCA, "la competencia objetiva que cabe atribuir a las Comunidades Autónomas en materia de defensa de la competencia se halla limitada a aquellas actuaciones ejecutivas que hayan de realizarse en el territorio de cada Comunidad Autónoma y que no afecten al mercado supra autonómico. Y, así, la competencia estatal se extiende no sólo a la normación sino también a todas las actuaciones ejecutivas en relación con aquellas prácticas que puedan alterar la libre competencia en el ámbito supra autonó-

\footnotetext{
${ }^{4}$ Anuario de la competencia 2003. ICO-Marcial Pons. Madrid 2004 p. 14.
} 
mico o en el conjunto del mercado nacional, aunque tales actuaciones se realicen en el territorio de una única Comunidad Autónoma". 5

Como sea que la teoría del efecto resulta muy conflictiva en su aplicación práctica y deja muchas zonas grises, se prevé también el recíproco intercambio de información entre órganos administrativos así como la creación de una Junta Consultiva paritaria de naturaleza arbitral y de un Consejo de Defensa de la Competencia (al que incidentalmente apuntamos convendría cambiar la denominación para no confundirlo con el Consejo CNC creado por la Ley $15 / 2007)$ para promover la aplicación uniforme de la normativa de defensa de la competencia.

La redacción del artículo 15 de la Ley 15/2007 establece, bajo la rúbrica de "Coordinación de la Comisión Nacional de la Competencia con los órganos competentes de las Comunidades Autónomas”, que:

1. La coordinación de la Comisión Nacional de Competencia con los órganos competentes de las Comunidades Autónomas se llevará a cabo según lo dispuesto en la Ley 1/2002, de Coordinación de las Competencias del Estado y las Comunidades Autónomas en materia de Defensa de la Competencia.

2. A efectos de facilitar la cooperación con los órganos jurisdiccionales y la coordinación con los órganos reguladores, la Comisión Nacional de Competencia y los órganos de defensa de la competencia de las Comunidades Autónomas, habilitarán los mecanismos de información y comunicación de actuaciones previstos en los artículos 16 y 17 de la presente Ley respecto de aquellos procedimientos que hayan sido iniciados formalmente según lo previsto en la Ley 1/2002, de 21 de febrero, de Coordinación de las Competencias del Estado y las Comunidades Autónomas en materia de Defensa de la Competencia.

Dichos mecanismos de cooperación y colaboración general e intercambio de información recíproca se concretan en varios aspectos:

- Los órganos autonómicos podrán aportar información o presentar observaciones a los órganos jurisdiccionales sobre cuestiones relativas a los casos de prácticas restrictivas y abusos de posición de dominio en los términos previstos en la LEC. (art. 16.2)

- Los autos de admisión a trámite de las demandas y las sentencias que se pronuncien en los procedimientos de aplicación de los arts. 81 y 82 TCE o de los arts. 1 y 2 de la LDG se comunicarán a la Comisión

\footnotetext{
5 NADAL, M. y ROCA, J. La descentralización de la política de defensa de la competencia en España: situación y perspectivas. Instituto de la Empresa Familiar. Barcelona, 2003, p. 48.
} 
Nacional de Competencia. Ésta habilitará los mecanismos de información necesarios para comunicar estas sentencias a los órganos autonómicos (art. 16.3.)

- La CNC remitirá a los órganos autonómicos una nota sucinta de las actuaciones practicadas de oficio y copia de todas las denuncias, respecto de las que existan indicios racionales de infracción, que se refieran a conductas que afecten su respectiva Comunidad Autónoma (DA $10^{a}$. Modificación de la Ley $1 / 2002$ )

- La CNC en el ejercicio de sus funciones, recabará del órgano autonómico, informe preceptivo, no vinculante, a emitir en el plazo de 20 días, en relación con las conductas que, afectando a un ámbito supra autonómico o al conjunto del mercado nacional, incidan de forma significativa en el territorio de la respectiva Comunidad Autónoma. (DA 10․․․ Modificación de la Ley $1 / 2002$ )

- La autoridad nacional remitirá también en los casos anteriores, copia del pliego de concreción de hechos, denuncia, pruebas practicadas, acuerdos y resoluciones. (DA $10^{\underline{a}}$. Modificación de la Ley 1/2002)

En definitiva, el modelo descentralizado desemboca en una doble red de autoridades estatales y autonómicas de defensa de la competencia que aplican la Ley de manera coordinada.

\subsection{Modelo descentralizado versus modelo participativo}

Frente a la más conocida coordinación administrativa, existe también la posibilidad de reconducir la pluralidad de Administraciones con competencia en una materia mediante la composición plural de los mismos órganos del Estado. En este caso, no estamos ante una técnica administrativa relacional sino ante una interpretación integradora de las previsiones constitucionales recogidas en el artículo 2 CE. ${ }^{6}$

Efectivamente, los órganos administrativos estatales de composición plural consiguen el mismo objetivo que la coordinación administrativa pero superan la dualidad de Administraciones que se mantienen en una situación de coordinación. La coordinación es una actuación administrativa externa a la propia organización mientras que la composición plural integra en la estruc-

\footnotetext{
${ }^{6}$ Vide al respecto BALLBÉ MALLOL, M y MARTÍNEZ QUIRANTE, R. Soberanía dual y constitución integradora. Ariel, Barcelona, 2003.
} 
tura orgánica las diferentes voluntades administrativas que pasan a manifestarse de forma única.

La cultura administrativa tradicional en España no contempla la composición como principio de actuación de las Administraciones públicas. Sin embargo, ello no debe llevarnos a pensar que la misma no tiene cabida en el diseño constitucional sino todo lo contrario. Mientras que la coordinación resulta un principio de organización administrativa, la composición y la integración son verdaderos principios de alcance constitucionales.

El modelo de co-participación de las Comunidades en las instituciones centrales se ha apuntado como causa determinante en algunos de los procesos de remodelación de estas instituciones u órganos administrativos, de los que podemos citar, el Instituto Español de Comercio Exterior (ICEX), el Consejo Asesor del Turismo y la reciente reforma de la Agencia Estatal de Administración Tributaria efectuada por la Ley 21/2001 de 27 de diciembre.

La propuesta de nuevo Estatuto de autonomía para Cataluña que redactó el Parlamento catalán imponía una solución que podemos llamar de integración participativa de los distintos niveles de defensa de la competencia en el plano interno. Así, el art. 154.4. preveía que la Generalitat participaría en los órganos estatales de defensa de la competencia (o sea CNC):

"La Generalitat participa en los organismos de carácter estatal y europeo que tienen atribuidas funciones homólogas en materia de promoción y defensa de la competencia”

Esta previsión ha desaparecido después del trámite parlamentario ante la Comisión Constitucional del Congreso de los Diputados y no aparece en la Ley Orgánica 6/2006 de 19 de julio por la que se aprueba el Estatuto de Autonomía de Cataluña. Como explica la diputada MALARET GARCÍA (PSOE) en la tramitación parlamentaria de la Ley 15/2007, "en el proyecto de Ley se amplían las competencias de los órganos autonómicos en su territorio, en línea con las nuevas atribuciones que se asignan a la autoridad nueva que se crea, la Comisión Nacional de Defensa de la Competencia. Pero en el marco actual, en coherencia con el marco constitucional español que resuelve de manera distinta al modelo federal alemán la concurrencia de competencias en materia económica, define un sistema de competencias excluyentes, en el que la competencia o es de la autoridad central o es de la autonómica. Se trata de un sistema en el que ambas autoridades están en el mismo plano formal o jurídico, recayendo la resolución de conflictos en la 
junta consultiva independiente. Este es el marco que tenemos y en el que se inserta el proyecto de ley"7.

Por tanto, la implicación de las Autonomías en la política de defensa de la competencia se articula a través de la descentralización y la coordinación de actuaciones y no a través de la participación en las instituciones nacionales. Tampoco era muy lógico que un Estatuto de Autonomía impusiera o predeterminara la naturaleza y composición de un organismo estatal.

La dicción actual del Estatuto de Autonomía de Cataluña es bastante parecida a la que contiene el Estatuto de Autonomía de Andalucía aprobado por la Ley Orgánica 2/2007 de 19 de marzo

"La Comunidad Autónoma de Andalucía asume competencias exclusivas en:

(...)

5‥ Promoción de la competencia en los mercados respecto de las actividades económicas que se realizan principalmente en Andalucía y el establecimiento y regulación de un órgano independiente de defensa de la competencia" (art. 58.1.)

"La Comunidad Autónoma asume competencias ejecutivas en:

(...)

5․ Defensa de la competencia en el desarrollo de actividades económicas que alteren o puedan alterar la libre competencia del mercado en un ámbito que no supere el territorio de Andalucia, incluidos la inspección y la ejecución del régimen sancionador" (art. 58.4)

La competencia varía de exclusiva a ejecutiva según se trate de promoción de la competencia o de defensa de la competencia. En ambos casos se utiliza de manera poco precisa el concepto de territorio donde se realicen principalmente las actividades cuando en realidad el criterio de conexión que ampara la intervención autonómica no es ni el territorio ni el lugar de ejercicio de la actividad sino los efectos económicos de la misma. Las Comunidades pueden intervenir en materia de defensa de la competencia cuando las prácticas que puedan alterar la libre competencia se efectúen en un ámbito interno de la Comunidad y sus efectos no se extiendan al conjunto del mercado nacional. Por lo demás, Andalucía cuenta en el artículo 58.1. y en el 164 con la habilitación para regular su organismo autonómico de defensa de la competencia, con reserva de Ley y con naturaleza de órgano independiente.

\footnotetext{
${ }^{7}$ Diario de Sesiones del Congreso de los Diputados. VIII Legislatura no 231, sesión plenaria del 8 de febrero de 2007.
} 


\subsection{Efectos de la descentralización sobre el conjunto de la defensa de la com- petencia}

La experiencia acumulada hasta la actualidad por el Tribunal y el Servicio de Defensa de la Competencia demuestran un progresivo aumento del número de expedientes resueltos. Este fenómeno provoca la sobrecarga de trabajo para una estructura administrativa bastante modesta en lo que a medios humanos y materiales refiere. Resulta interesante reflexionar sobre los efectos de la descentralización sobre la futura carga de trabajo de la CNC. Así, en el futuro puede pensarse en un reparto de casos entre autoridades centrales y autonómicas de manera que se liberen recursos humanos para la CNC.

Por el momento, "no es posible acreditar en 2003 un predominio de expedientes de ámbito autonómico. En consecuencia, atendiendo además al lento despliegue de los órganos autonómicos, los órganos estatales de defensa de la competencia no pueden confiar a corto plazo en una descarga apreciable de expedientes, que les permita acortar el tiempo de resolución e incrementar las labores de investigación de oficio". 8 Tampoco es demasiado halagüeña en este sentido la amplitud de los criterios de conexión de la Ley 1/2002 que hace difícil encontrar casos que correspondan al ámbito competencial de las autoridades autonómicas de la competencia.

En otro orden de cosas, resulta también interesante preguntarse por el alcance que la creación de un único órgano -Comisión Nacional de Competencia- va a suponer para la organización administrativa de las Comunidades. En principio, debe regir la potestad de autoorganización administrativa de las Comunidades en ejercicio de las competencias ejecutivas que les reconoce el ordenamiento. Esta visión se confirma si consideramos que la Disposición Final primera de la Ley 15/2007 establece que ésta se dicta al amparo de la competencia exclusiva del Estado ex art. 149.1.13. (ordenación económica) y no en cambio ex artículo 149.1.18 (bases del régimen de las Administraciones públicas). Además, la Disposición Adicional octava prevé que las referencias a la CNC hechas en la Ley se entenderán realizadas a los órganos correspondientes de las Comunidades Autónomas con competencia en la materia. A nuestro modo de ver, ni una ni otra disposición permiten entender la extensión obligatoria del modelo organizativo estatal a las Comunidades.

\footnotetext{
${ }^{8}$ ARZOZ SANTISTEBAN, X. "El impacto de los puntos de conexión de la Ley 1/2002 en la aplicación descentralizada del derecho de la competencia: análisis de las resoluciones adoptadas en 2003 por el TDC" Anuario de la Competencia 2003. ICO-Marcial Pons, Madrid, 2004, p. 340.
} 
De todas formas, como ya hemos tenido ocasión de expresar, ${ }^{9}$ la configuración de algunas de las funciones de los órganos de defensa de la competencia hace imprescindible la adopción de una estructura administrativa que garantice la independencia. Así, por ejemplo, en el ejercicio de la competencia de informe sobre las ayudas públicas o más todavía en el caso de la competencia sobre impugnación de actos y disposiciones administrativas de carácter general que puedan restringir la libre competencia, la independencia del organismo de competencia con respecto a la Administración general resulta imprescindible. En otras palabras, algunas de las nuevas competencias sustantivas que prevé la Ley 15/2007 sólo son realizables con un diseño institucional que garantice la independencia de las instituciones de defensa de la competencia.

3. LA LEY DEL PARLAMENTO DE ANDALUCÍA 6/2007, DE 26 DE JUNIO, DE PROMOCIÓN Y DEFENSA DE LA COMPETENCIA EN ANDALUCÍA

Andalucía ha sido la Comunidad que más recientemente se ha incorporado al fenómeno descentralizador en materia de defensa de la competencia con la aprobación por parte del Parlamento de Andalucía de la Ley 6/2007 de 26 de junio, de Promoción y Defensa de la Competencia en Andalucía. ${ }^{10}$

La norma regula el entramado institucional de la defensa de la competencia en Andalucía, poniendo especial énfasis en asegurar la independencia del órgano administrativo (art. 1.3.) como mecanismo de garantía de la eficacia de la política de control de los mercados. Así, el preámbulo de la Ley indica que "la Comunidad Autónoma de Andalucía, mediante la presente Ley, establece un modelo que, con la finalidad de mejorar el funcionamiento competitivo de los mercados, refuerza la independencia del órgano de control y la eficacia y eficiencia de los mecanismos de defensa de la competencia en Andalucía, creando un organismo autónomo especializado e independiente con capacidad jurídica pública diferenciada”.

\footnotetext{
${ }^{9}$ PADRÓS REIG, C. "Los organismos autonómicos de Defensa de la Competencia. Balance y perspectivas." Inédito 2007.

${ }^{10}$ BOJA no 131, de 4 de julio de 2007.
} 
Se crea así, la Agencia de Defensa de la Competencia de Andalucía (ADCA), como organismo autónomo de carácter administrativo que queda adscrita a la Consejería de Economía. La Agencia goza de personalidad jurídica pública diferenciada, plena capacidad jurídica, así como patrimonio y tesorería propios y en el ejercicio de sus funciones actuará con plena independencia. Las funciones de la Agencia se recogen en el artículo 3 de la Ley 6/2007.

La Agencia se compone de 5 órganos:

- la Dirección-gerencia

- el Consejo de Defensa de la Competencia

- el Departamento de Investigación

- el Departamento de estudios, análisis de mercados y promoción de la competencia

- la Secretaría general

La Dirección funciona como representante legal de la Agencia y tiene atribuidas funciones básicamente de dirección administrativa respecto a la contratación, personal, presupuesto, memoria anual y cuentas. Corresponde a la Gerencia la aprobación de las normas internas de funcionamiento en las cuales se establecerá su régimen administrativo y la organización de sus servicios y órganos (art. 10.2.d.) La persona que ocupe esta Dirección o gerencia administrativa será nombrada y separada por la Junta sin más requisitos, ni de tiempo ni de forma, a propuesta de la persona titular de la Consejería competente en materia de economía (art. 11).

Dado que la Dirección-gerencia se encarga de cuestiones de tanta relevancia como son la representación del organismo; la asistencia en representación de Andalucía a las reuniones del estatal Consejo de Defensa de la competencia o; la aprobación de la memoria anual, tal vez hubiera sido oportuno que el legislador contemplara algún tipo de hearing sobre la figura por parte del Presidente o del Consejo. A modo de ejemplo, el anteproyecto de la Ley 15/2007 LDC preveía que el gobierno oiría al Presidente CNC en el proceso de nombramiento del Director de Investigación de la Comisión. Aunque éste no fue el sistema finalmente adoptado, demuestra que debería existir una cierta comunicación entre los varios órganos de la Agencia para facilitar la unidad interna ${ }^{10 \mathrm{bis}}$.

El único mecanismo de contacto entre la gerencia y el Consejo, se prevé en el extraño caso de sustitución por vacante, ausencia o enfermedad del Pre-

10bis Esta comunicación se articula precismante mediante la Comisión de Coordinación prevista en los arts. 21 y 22 de los Estatutos de la Agencia aprobados por el Decreto 289/2007 de 11 de diciembre (BOJA no 245 de 14 de diciembre 2007). 
sidente. En esos casos le sustituirá alguno de los vocales del Consejo. También en caso de faltar dos miembros del Consejo, el gerente puede sustituir a uno de los vocales (art. 13.3 in fine.). Se trata de una previsión algo extraña puesto que la configuración y funciones de uno (Gerente) y otro (Vocales) son completamente distintas. Así se exigen requisitos de profesionalidad para los vocales pero no para la Dirección.

Además, la sustitución termina de enredarse en los artículos 11.2 y 13.1. Según esto, el Presidente del Consejo puede sustituir al Director-Gerente, pero en cambio, la Presidencia del Consejo nunca puede ocuparse por la persona titular de la Dirección-Gerencia (sí en cambio, una vocalía).

Junto con este órgano administrativo o gestor dentro de la Agencia, aparece también el Consejo. Está compuesto por un Presidente y dos vocales con función resolutoria de los asuntos que conozca. Se exige en estos casos un determinado perfil profesional y el mandato se determina en 5 años renovables una sola vez. Corresponden al Consejo todas las funciones y facultades de iniciación y resolución de procedimientos regulados en la normativa estatal de defensa de la competencia, relativos a actividades económicas que, sin afectar a un ámbito territorial más amplio que el de la Comunidad Autónoma de Andalucía, alteren o puedan alterar la libre competencia.

Las funciones del Presidente del Consejo son las ordinarias de un Presidente de un órgano colegiado (art. 17): convocar a iniciativa propia o a petición de cualquiera de las vocalías el Consejo y presidirlo; establecer el criterio de distribución de asuntos; mantener el buen orden y gobierno del Consejo; dar cuenta de las vacantes que se produzcan; delegar en la vocalías aquellas funciones que considere conveniente.

Las figuras del Presidente y del Gerente pueden dar lugar a una cierta tensión en el liderazgo de la Agencia. Si bien es verdad que la representación legal y la dirección corresponden al Gerente, la competencia por razón de la materia y las facultades resolutorias se sitúan en el Presidente. Si se permite la comparación, al igual que sucede en ciertas infraestructuras culturales, se divide entre director técnico y director artístico, con las tensiones que ello acarrea.

Si la resolución se atribuye al Consejo, la instrucción en cambio se encarga al Departamento de Investigación. Éste queda integrado dentro de la ADCA pero se garantiza la necesaria separación entre instrucción y resolución al tratarse de órganos distintos dentro de la misma estructura administrativa. El Departamento de Investigación es el órgano que ejerce las funciones de ins- 
trucción, investigación y vigilancia a las que se refiere la normativa estatal reguladora de la defensa de la competencia. No existen en la Ley condiciones especiales para el nombramiento del Director o Directora del Departamento de Investigación. ${ }^{11}$ Según el art. 19.1. "La persona titular de la Dirección del Departamento de Investigación de Defensa de la Competencia de Andalucía ostentará la jefatura del mismo, y ejercerá todas las competencias que la presente Ley atribuye a este Departamento, así como las facultades que establezcan los Estatutos de la Agencia y demás normativa de aplicación, y las que le sean delegadas".

Resulta interesante también abordar la relación del Director o Directora de Investigación con otros órganos de la Agencia. En principio, el Director tiene autonomía en la selección de los casos que deben ser objeto de expediente por parte de la Agencia. A pesar de ello, se contempla como competencia del Consejo la posibilidad de "interesar la instrucción de expedientes por el Departamento de Investigación”. Esta facultad puede comprometer la necesaria independencia entre la función instructora y la juzgadora. No resulta lógico que el Consejo que ha solicitado la persecución de una conducta tenga que determinar después el carácter de la misma. Esta cuestión, sin embargo, no se presenta exclusivamente en el modelo organizativo andaluz.

Junto con la relación Director de Investigación-Consejo, aparece también regulada la relación entre Director de Investigación y Director-Gerente. Efectivamente, prevé el artículo 18.2. prevé que el Departamento de Investigación deba "elevar a la Dirección-Gerencia de la Agencia las propuestas para realización de comunicaciones y notificaciones reguladas en el artículo 2 de la Ley 1/2002, de 21 de febrero". En principio no se trata más que de dar trámite de esas cuestiones a través del órgano administrativo que maneja las relaciones externas de la Agencia. No existiría por tanto la posibilidad de un control de fondo del Gerente sobre las actividades del Director o Directora de Investigación.

Se regula también en la Ley de manera muy interesante y novedosa la existencia de un Departamento de estudios, análisis de mercados y promoción de la competencia. La promoción de la competencia (competition advocacy), es

${ }^{11}$ El Consejo Económico y Social de Andalucía advierte al respecto que: "Consideramos conveniente que el Director del Departamento pudiera contar con un Adjunto experto, que lo asesorara en la emisión de sus decisiones, así como que fuera nombrado entre juristas o economistas de reconocido prestigio" Dictamen CES Andalucía no 7/2006 sobre el Anteproyecto de Ley de Promoción y Defensa de la Competencia de Andalucía. 8 mayo 2006. Entre las funciones de la Dirección-Gerencia previstas en los Estatutos, se halla la de nombrar y separar tanto al Director/a de Investigación como al Secretario/a General. 
una función altamente importante de las autoridades de defensa de la competencia por cuanto contribuye a difundir y transmitir los valores y los beneficios que esta política pública aporta a los mercados y a los ciudadanos. La Ley estatal 15/2007 ha sido objeto de nuestras críticas al desaprovechar la oportunidad de crear un órgano específico para esta función, cosa que sí hace, en cambio, la legislación andaluza. Corresponden a este Departamento de estudios las funciones relativas a:

- realización de estudios de competencia y análisis de mercados dirigidos a promover la competencia

- tareas de información, asesoramiento y propuesta

- elaboración de la memoria anual de la Agencia ${ }^{12}$

- asistencia a la Gerencia en las labores de colaboración con otros organismos

- asistencia al Consejo en la elaboración de informes y dictámenes

Además, se regula en un artículo separado el carácter público de las resoluciones y demás actos y actuaciones en materia de defensa de la competencia, (art. 6.1.) lo que debería incluir los informes y estudios. Corresponde también al Departamento de Investigación la gestión del Sistema de Información de Promoción y Defensa de la Competencia de Andalucía y, en particular, la instrumentación de la publicidad de las actuaciones de la Agencia.

Finalmente, la Ley regula la Secretaría General (art. 21). Corresponde a la misma la administración del presupuesto y el régimen interno de la Agencia. Sus funciones se centran en:

- prestar asistencia y colaboración al Consejo en el cumplimiento de las funciones que le son propias y, en concreto, en relación con la tramitación de los procedimientos en materia de defensa de la competencia

- velar por la correcta y adecuada ejecución de las resolución del Consejo de Defensa de la Competencia de Andalucía, así como elevarle periódicamente los informes relativos al grado de cumplimiento

- elaborar los informes sobre los anteproyectos y proyectos de normas de la Junta de Andalucía que incidan en la libre competencia

- llevar a efecto la gestión de los asuntos relacionados con el personal y las generales de administración, registro y archivo

\footnotetext{
${ }^{12}$ Memoria que, sin embargo, debe aprobarse por la Gerencia.
} 
- asistir a la Dirección-Gerencia de la Agencia en la elaboración del borrador del anteproyecto de presupuesto y en la ejecución del mismo

- elaborar las cuentas anuales de la Agencia

- $\quad$ ejercer aquellas facultades que le atribuyan los Estatutos de la Agencia, y demás normativa de aplicación, así como las que le sean delegadas.

Las competencias de la Secretaría General presentan ciertas duplicidades con las de la Dirección-Gerencia, con la que deberá a buen seguro coordinarse. Por ejemplo, las cuentas anuales serán elaboradas por la Secretaría y aprobadas por la Dirección. Lo mismo puede decirse de la gestión de personal. Igual de problemáticas pueden presentarse el resto de funciones. Así, la de elaborar informes sobre normas y proyectos de normas que afecten a la libre competencia parece que debería ser una función atribuida al órgano materialmente competente en las cuestiones de fondo (Consejo) y no a una secretaría, en tanto que asistente jurídico. Además, otra de las funciones, la relativa a la vigilancia del grado de cumplimiento de la ejecución de las resoluciones, podría englobarse dentro del ámbito de actuación del Departamento de Investigación que es quien tiene potestades de investigación, requerimiento e intimación, mucho más útiles para el desarrollo de la función. Por ende, a quien corresponde la función de vigilancia (también de la ejecución de las resoluciones) es, según la dicción del art. 18.1, el Departamento de Investigación. En definitiva, la regulación de la Secretaría General presenta elementos susceptibles de una mayor clarificación y mejora.

Finalmente, aunque no aparezca en el elenco de funciones, corresponde a la Secretaría General asistir a las reuniones del Consejo (con voz pero sin voto). La función de la Secretaría General en el Consejo puede ser sustituida por el Director o Directora de Estudios en caso de ausencia o enfermedad.

La constitución efectiva de la Agencia tendrá lugar en el momento de entrada en vigor de sus Estatutos que deberán ser aprobados por el Consejo de Gobierno de la Junta antes del 5 de enero de $2008^{12 b i s}$.

12 bis Los Estatutos fueron aprobados por Decreto 289/2007 de 11 de diciembre (BOJA no 245 de 14 de diciembre 2007) y la fecha de inicio efectivo se determinará mediante Orden del Consejero en un plazo máximo de 2 meses. 
4. SIMILITUDES Y DIFERENCIAS ENTRE LA LEY ESTATAL 15/2007, DE 3 DE JULIO, DE DEFENSA DE LA COMPETENCIA Y LA LEY ANDALUZA 6/2007, DE 26 DE JUNIO, DE PROMOCIÓN Y DEFENSA DE LA COMPETENCIA DE ANDALUCÍA

\subsection{Autoridad única: Comisión Nacional de Competencia y Agencia de De- fensa de la Competencia de Andalucía}

Uno de los aspectos más visibles de la Ley 15/2007, de 3 de julio, es la sustitución de la estructura dual existente hasta la actualidad entre Servicio de Defensa de la Competencia (SDC) y Tribunal de Defensa de la Competencia (TDC) por un único órgano: la Comisión Nacional de Competencia. Sin embargo, la necesidad de garantizar la separación entre la función instructora y la enjuiciadora de los expedientes se mantiene inalterada en el seno de la propia Comisión. Así, la estructura interna de la CNC divide entre Consejo (antiguo TDC) y Dirección de Investigación (antiguo SDC), optando pues el legislador por una estructura piramidal-coordinada y no vertical-jerárquica. Lo mismo puede decirse de la Agencia andaluza que bajo la misma institución agrupa tanto al Consejo como al Departamento de Investigación.

Mientras que el Consejo CNC es el órgano colegiado de resolución, la Dirección de Investigación realiza las funciones de instrucción de expedientes, investigación, estudio y preparación. La diferencia fundamental puede residir no tanto en la desaparición de la dualidad orgánica sino en el carácter más piramidal del nuevo diseño institucional. En la actualidad, la separación entre SDC y TDC era absoluta mientras que en el nuevo modelo, el nombramiento del Director de Investigación debe contar con la aprobación por mayoría simple del Consejo. ${ }^{13}$

La Agencia andaluza se diferencia en este punto de la estatal puesto que no hay ninguna intervención en el nombramiento del titular del Departamento de Investigación. En la redacción inicial del Proyecto estatal, el Director era nombrado por el gobierno con la sola previsión de la consulta previa al Presidente. Ello provocaba, pues, es cierto mantenimiento de la dinámica dual puesto que la disociación entre función instructora y función enjuiciadora se arrastraba al propio diseño institucional a través del mantenimiento de legitimidades diversas. Este aspecto ha sido modificado en el redactado final que prevé

\footnotetext{
13 Este aspecto ha sido introducido en la tramitación parlamentaria, suponiendo un cambio muy relevante respecto al texto del proyecto.
} 
ahora la aprobación del nombramiento del DI por mayoría simple del Consejo $\mathrm{CNC},{ }^{14}$ pero se mantiene inalterado en la Ley andaluza 6/2007.

Igualmente, en el caso estatal, el conjunto de la CNC se sitúa bajo la dirección del Presidente, mientras que la Agencia andaluza introduce la figura del Director-Gerente. Ello rebaja en cierta manera el perfil del Presidente que de ser el máximo órgano de la institución pasa a ser el presidente de un órgano colegiado (Consejo ADCA).

Al hilo de lo anterior, es destacable como la adopción del modelo piramidal permite tener más influencia sobre la instrucción de los expedientes. Así una de las críticas comunes al sistema institucional vigente era el hecho que la dependencia del SDC del Ministerio de Economía permitía regular el flujo de casos que examinaba el TDC. Si bien existía independencia en la resolución, ésta disminuía en la selección de casos. Ahora, la Ley 15/2007 contempla entre las funciones del Presidente CNC el impulsar la actuación inspectora (artículo 32.d); elaborar planes de actuación (artículo 32.d); coordinar el Consejo con la Dirección (artículo 32.e). Estos elementos no existen para el modelo andaluz con lo cual, la crítica del antiguo sistema estatal (falta de independencia en la selección de los casos), se continuará dando.

En Derecho comparado parece prevalecer el modelo administrativo de institución única. Así, la misma UE constituye junto con Alemania, Italia o Portugal, ejemplos de esta modalidad. La adopción de un modelo de institución única parece reforzar la necesidad de la independencia de la institución puesto que desaparece el equilibrio de legitimidades que representaba la dualidad orgánica entre SDC y TDC. Como indica el ex presidente del Tribunal GONZALO SOLANA, "a nivel internacional se observa recientemente una convergencia en la mayoría de sistemas de los Estados Miembros de la Unión Europea hacia un modelo de una sola institución administrativa encargada tanto de la instrucción como de la resolución de los expedientes sancionadores. Actualmente, Alemania, Dinamarca, Holanda, Irlanda, Italia, Portugal, Reino Unido y Suecia cuentan con un modelo institucional de defensa de la competencia de estas características. Dentro de los quince antiguos Estados Miembros sólo Bélgica, Francia y España permanecen con un modelo basado en dos instituciones". ${ }^{15}$

14 cfr. Enmienda nº 100 del Grupo Parlamentario Popular en el Congreso. BOCG Serie A nº $100-18$, de 26 de febrero de 2007.

15 SOLANA GONZÁLEZ, G. "Pasado, presente y futuro del modelo español de defensa de la competencia” Anuario de la Competencia 2003. ICO-Marcial Pons, Madrid, 2004 p. 50. 
En definitiva, la unidad orgánica se ha acompañado, en el modelo estatal, con una mayor independencia del conjunto, mientras que en Andalucía se predica sólo una independencia formal de la Agencia sin mecanismos de garantía de la misma.

La doctrina ha destacado entre las virtudes del modelo dual su capacidad para brindar una mayor protección de los derechos de defensa de las partes en un procedimiento. ${ }^{16} \mathrm{En}$ un diseño con dos instituciones, existe siempre la posibilidad de revisión en vía administrativa de los acuerdos adoptados a través del actual recurso contra los actos de SDC. Este derecho de defensa es especialmente importante cuando existe un alto grado de discrecionalidad técnica en el asunto y los operadores no conocen de antemano los criterios normativos. Dicho en otras palabras, la inexistencia de reglas claramente codificadas y la decisión casuista son elementos para considerar a favor del mantenimiento de una dualidad de instituciones. Según la OCDE, algunos de los derechos de defensa que no deben perjudicarse con la unificación orgánica son: el derecho de acceso a la Agencia durante el procedimiento para poder conocer la calificación administrativa de los actos; el derecho de audiencia en un procedimiento justo y previo a la decisión; el derecho a recibir una notificación de la resolución debidamente motivada; así como el derecho de recurso ante la jurisdicción ordinaria. Por ello, propugnamos que la unificación orgánica que suponen tanto la CNC como la ADCA debería acompañarse de una mayor transparencia en los procedimientos y una mayor codificación de las enforcement guidelines. ${ }^{17}$

Con estos dos aspectos en tensión (unidad e independencia; unidad y seguridad) se demostraría la conexión entre diseño institucional y entorno normativo. La nueva Comisión no sólo implica una reforma administrativa sino un replanteamiento más reformador de la política de defensa de la competencia, menos politizada y más transparente y predecible. ${ }^{18}$ No es así en el modelo adoptado por el legislador andaluz en la Ley 6/2007.

\footnotetext{
${ }^{16}$ WILS, W.P. Principles of European Antitrust Enforcement. Hart Publishing, Londres, 2005. También SOLANA GONZÁLEZ, G. para quien "la Ley, al separar las fases de instrucción y resolución de los expedients en organismos distintos y ubicar al Tribunal en una posición de órgano de revisión de las actuaciones del Servicio, ha sabido crear un balance institucional adecuado". SOLANA GONZÁLEZ, G. "Unas bases sólidas para acometer la reforma" Anuario de la Competencia 2004. ICO-Marcial Pons, Madrid, 2005.

${ }^{17}$ Comunicaciones CNC en dicción de la DA $3^{\text {a }}$ de la Ley 15/2007.

${ }^{18}$ El Ministro de Economía define las reformas en los siguientes términos: "El proyecto plantea las siguientes reformas: reforzar la independencia en la toma de decisiones, y como conse-
} 


\subsection{Naturaleza jurídica}

El TDC pierde su carácter de Organismo Autónomo ${ }^{19}$ que se extingue y la CNC se configura como un nuevo Organismo Público. ${ }^{20}$ Los Organismos Autónomos dependen de un Ministerio al que corresponde la dirección estratégica, la evaluación y el control de los resultados. En cambio, los organismos públicos especiales ex Disposición Adicional 10 $\mathrm{a}$ LOFAGE, gozan de una mayor autonomía puesto que:

- $\quad$ su ley de creación les puede reconocer expresamente independencia funcional o especial autonomía

- $\quad$ se rigen por su legislación específica

- el gobierno no ostenta una competencia general de dirección ni evaluación

- el gobierno debe ejercer sus facultades con respeto al ámbito de autonomía otorgado por la normativa específica

En los demás aspectos (personal, bienes, contratación y presupuesto) su funcionamiento es esencialmente idéntico a los Organismos Autónomos, con lo que la reforma no tendría especial trascendencia en esos aspectos.

El cambio de naturaleza supone, pues, un reforzamiento de la independencia de la CNC que viene a unirse a la caracterización administrativa de las llamadas Administraciones Independientes, como la Comisión Nacional del Mercado de Valores, la Agencia de Protección de Datos, la Comisión Nacional de la Energía o la Comisión del Mercado de Telecomunicaciones, por nom-

cuencia la transparencia y responsabilidad frente al mundo empresarial y a la sociedad en general; en segundo lugar, reforzar la seguridad jurídica de los operadores económicos y la agilidad y eficacia administrativa, especialmente, en el caso de las conductas restrictivas; finalmente, dotar al sistema de una mayor eficiencia al ampliar el número de organismos que aplican la Ley. El proyecto de ley prevé la cooperación con los órganos jurisdiccionales, la coordinación con los reguladores sectoriales y la colaboración con la Comisión Europea y las autoridades de competencia de otros Estados miembros, todo ello respetando el esquema de distribución de competencias entre Administración central y comunidades autónomas, de acuerdo con lo dispuesto en la Ley 1/2002" Diario de Sesiones Congreso de los Diputados. VIII legislatura no 231 de 8 de febrero de 2007.

${ }^{19}$ Artículo 43.1. a) de la Ley 6/1997, de 14 de abril, de Organización y Funcionamiento de la Administración General del Estado, (LOFAGE).

${ }^{20}$ Disposición Adicional 10 $0^{\mathrm{a}}$ de la Ley 6/1997, de 14 de abril, de Organización y Funcionamiento de la Administración General del Estado, (LOFAGE). 
brar algunas de las que expresamente se recogen en la citada DA $10^{\underline{a}}$ de la Ley 6/1997 LOFAGE, que se modifica ahora para incluir expresis verbis la Comisión Nacional de Competencia.

En el caso andaluz, se trata sólo de una Agencia que se configura como un organismo autónomo de carácter administrativo que está adscrito a la Consejería competente en materia de economía. Es cierto que la Agencia actuará con autonomía orgánica y funcional y plena independencia de las Administraciones públicas, pero por la propia configuración como organismo autónomo se mantiene el control de eficacia sobre la actividad, potestad típica de los Organismos Autónomos pero no de las Administraciones independientes. ${ }^{21}$ Además, la independencia de las agencias administrativas, en general, puede perseguirse mediante los procedimientos de nombramiento de los miembros y/o mediante el control parlamentario ex ante (líneas básicas de actuación) y ex post (mediante la rendición de cuentas). En Derecho comparado, los modelos de institución única garantizan su independencia mediante el nombramiento por parte de las asambleas legislativas (Italia) o mediante la rendición de cuentas al Parlamento y un mandato superior al de una legislatura (Alemania). En la nueva Ley andaluza parece prevalecer modelo de unidad orgánica pero no se articula prácticamente ningún mecanismo de independencia: no hay hearing parlamentario previo al nombramiento de los Vocales; no hay mecanismos de rendición de cuentas ante la Asamblea, etc. Lo único que existe es un mandato de 5 años que por definición es superior al del ciclo político-electoral.

No cabe duda que la transformación más importante la experimenta el extinto SDC. La nueva Dirección de Investigación pasará a ser un órgano especializado de una Administración independiente, abandonando su carácter de órgano administrativo jerarquizado en el seno del Ministerio de Economía. A pesar de ello, las condiciones de nombramiento del Director de Investigación presentaban algunos interrogantes que fueron objeto de severas críticas por parte del Consejo de Estado en el Dictamen 1080/2006 de 20 de julio de 2006, relativo al Anteproyecto de Ley de Defensa de la Competencia. Según el

${ }^{21}$ El Consejo de Estado advertía ya sobre el particular en su Dictamen 1080/2006 de 20 de julio de 2006: "El artículo 19.1 del anteproyecto señala que la Comisión Nacional de Competencia está adscrita al Ministerio de Economía y Hacienda, que ejercerá el control de eficacia sobre su actividad. Puesto que ya existen otro tipo de controles como el económico y financiero (artículo 22.3) o, singularmente, el parlamentario (artículo 28), parece innecesario incluir una previsión como la que ahora se cuestiona. De mantenerse habría que precisar que dicho control no puede ejercerse en detrimento de la autonomía de la institución." 
alto órgano consultivo: "Según el artículo 29.4 del anteproyecto, el Director de Investigación será nombrado por el Gobierno mediante Real Decreto, a propuesta del Ministro de Economía y Hacienda y previa consulta al Presidente de la Comisión. Si uno de los medios para garantizar la independencia de la institución es sacar las facultades de instrucción del ámbito del Ministerio de Economía y Hacienda, carece de sentido que quien ostenta la jefatura de la unidad encargada de la instrucción sea objeto de nombramiento discrecional por el Gobierno y a propuesta del Ministro, sin exigirse además una cualificación específica alguna para desempeñar ese puesto. Por ello, se considera necesario que el nombramiento del Director de Investigación se efectúe por el propio órgano, mediante propuesta vinculante aprobada por el Consejo y con la exigencia de concretos requisitos de cualificación profesional apropiados a la naturaleza del puesto a desempeñar."

Esos interrogantes han sido felizmente despejados en la tramitación parlamentaria que ha transformado el sistema de nombramiento del Director de Investigación de la simple consulta previa al Presidente CNC a la aprobación del candidato por mayoría simple del Consejo CNC. En el caso andaluz, en cambio, nada se dice sobre las condiciones del candidato a la dirección del Departamento de Investigación, ni en cuanto a sus requisitos, perfil o sistema de nombramiento.

En el informe World Development Report 2002 del World Bank se menciona que el 63\% de los países examinados cuentan con autoridades de competencia que son independientes de un Ministerio. Según el Global Competition Forum de la OCDE, "la observación más interesante del material de cuestionario es la gran variedad de modelos diferentes y soluciones para los diseños institucionales. El estatus de la autoridad de competencia en la Administración pública puede variar desde la independencia estructural del gobierno hasta la integración en la estructura ministerial, sin predominio evidente de ninguno de los enfoques. La implicación en las funciones del gobierno también varía ampliamente, desde el Presidente de autoridad de competencia teniendo un asiento en el gabinete de gobierno a las autoridades teniendo nula representación en cualquier nivel político. Lo que es particularmente chocante, y algo inconsistente con las respuestas al cuestionario sobre los objetivos de la política de competencia, es el grado en que virtualmente todos los destinatarios del cuestionario, independientemente del diseño institucional de la agencia de competencia en su jurisdicción, se consideran total o altamente independiente de influencia política en la aplicación del derecho de la competencia”. ${ }^{22}$

\footnotetext{
22 OEDC Global Forum on Competition. Optimal design of a competition authority" 3 feb. 2003. CCNM/GF/COMP(2003)2, p. 6.
} 


\subsection{Responsabilidad y rendición de cuentas}

La defensa de la competencia no es una opción fácilmente objetivable por lo que debe contemplarse como un proceso más que como un acto. Un proceso debe adaptarse constantemente a las cambiantes condiciones de incertidumbre donde se aplica la política pública. A modo de ejemplo, no siempre es fácil aventurar qué nivel de integración vertical en un determinado sector puede dañar la competencia o en qué casos la ostentación de una legítima posición de dominio puede desembocar en conductas anticompetitivas por el propio estrangulamiento del sector (los llamados bottlenecks). En todos estos casos, a pesar de ser la defensa de la competencia una cuestión altamente tecnificada, impone opciones reguladoras no siempre neutras. Cuando la función de los órganos de defensa de la competencia es la regulación de los mercados mismos (o su recreación en situaciones post-monopolistas), el margen de independencia puede verse reducido puesto que la función se asemeja más a la clásica política económica. Cuando las autoridades de competencia determinan el grado de concentración o la existencia de una posición de dominio que ponga en peligro la competencia efectiva en el mercado, o la exención a ciertas categorías de restricciones, existen razones para considerar que la función es casi-política.

Por ello, la independencia debe acompañarse de mecanismos de responsabilidad ante la sociedad: evaluación ex post de las medidas; publicación estadística de los casos; motivación suficiente de las decisiones; revisión constante del conocimiento disponible y; cantidad y calidad de los medios humanos encargados de la institución, entre otros. Todos estos elementos, calificados como fundamentales por la US Trade Commission pueden hallarse en cierta medida en los artículos 27 y 28 de la Ley 15/2007 que decididamente aumenta la publicidad de las actuaciones de las autoridades españolas de defensa de la competencia.

Por una parte, el Presidente de la Comisión elaborará un Plan de actuación donde se fijarán las prioridades para el futuro. El Consejo aprueba asimismo la memoria anual del organismo y los planes anuales y plurianuales de actuación en los que se definen objetivos y prioridades (art. 34.10). Todos estos elementos son objeto de presentación y discusión ante la Comisión de Economía y Hacienda del Congreso de los Diputados. Igualmente, la Comisión Nacional de la Competencia enviará al Ministro de Economía y Hacienda y a la Comisión de Economía y Hacienda del Congreso de los Diputados sus informes anuales sobre la situación competitiva de los mercados y sobre la actuación del sector público así como los informes sectoriales que apruebe. 
Ninguno de estos elementos de responsabilidad y rendición de cuentas se halla presente en la actividad de la Agencia de la Defensa de la Competencia de Andalucía. Si algo, el artículo 6.2. establece que la Agencia hará pública una memoria anual de actuaciones que se enviará a la persona titular de la Consejería competente en materia de economía y a la Comisión competente en materia de economía del Parlamento de Andalucía. Comparativamente con la regulación estatal, la regulación andaluza de la rendición de cuentas aparece como formalista e insuficiente.

\subsection{Miembros, personal y régimen económico}

Para la Comisión Nacional, los miembros y su Presidente se nombran por el gobierno previa comparecencia parlamentaria. ${ }^{23}$ Se reforma la duración antigua del mandato $\left(5+5\right.$ años) a un único mandato de 6 años. ${ }^{24}$ Se separa el ciclo electoral y político de la política de defensa de la competencia a la vez que se produce una significativa reducción del mandato total de los consejeros. Hasta ahora podían permanecer en su cargo 10 años y con la reforma sólo 6. Ello aumentará la rotación de miembros en el seno de la CNC.

La duración de los mandatos oscila entre dos opciones: un mandato corto con posibilidad de renovación (por una o más veces) o un mandato largo sin posibilidad de renovación. A mayor duración del mandato, mayor refuerzo a la independencia de los consejeros. Así, el paradigma podría ser el Bundeskartellamt con mandatos indefinidos para ciertos cargos. ${ }^{25}$ Cuando el mandato es excesivamente corto y no existe posibilidad de renovación puede producirse la patología conocida como revolving door. Si el Consejero termina su mandato con una edad relativamente joven, puede verse obligado a pensar más en su futuro profesional que en las funciones inherentes a la defensa de la competencia. Así, el caso español opta por un mandato relativamente breve y la no renovación. Si bien ello aleja al Consejero de la influencia política, puede favorecer cierta captura por los intereses de la industria. No se justifica tampoco porque no se adopta una duración de mandato algo más extensa.

\footnotetext{
${ }^{23}$ No así, en cambio, para el Director de Investigación.

${ }^{24}$ Según establece el artículo 29.3 el mandato no es renovable.

25 Sobre el sistema alemán, vide, CASES PALLARÉS, Ll. Derecho administrativo de la defensa de la competencia. Marcial Pons. Madrid, 1995.
} 
En la Agencia andaluza, se mantiene invariable el mandato de 5 años renovable por una sola vez. No existe ninguna previsión de período obligatorio de cool-off o cuarentena al abandonar el cargo, aspecto, en cambio que se incluye en la redacción definitiva del texto normativo del Estado, incorporándose un apartado 2 al artículo $31 .{ }^{26}$

Además, algunos autores coinciden también en que un mayor nivel de rotación en los puestos puede considerarse como un indicador negativo de independencia. ${ }^{27}$

En cuanto al régimen económico, la CNC goza también de cierta independencia económica: elabora y aprueba su propio presupuesto (con la estructura que fije el Ministerio de Economía) que se eleva al Gobierno. El presupuesto CNC integra el Presupuesto General del Estado. Los medios económicos de la $\mathrm{CNC}$ son: las asignaciones presupuestarias, su patrimonio propio y los ingresos obtenidos por la liquidación de tasas devengadas por la prestación de servicios derivados del ejercicio de sus competencias (particularmente la tasa por análisis y estudio de las operaciones de concentración).

En cambio, la Agencia andaluza, a pesar de gozar de la misma estructura en cuanto a sus recursos (art. 4.3.), no dispone de la misma autonomía financiera por cuanto la generación de ingresos propios de su actividad no cuenta con la tasa por control de concentraciones puesto que no le corresponde esta competencia. A igualdad de estructura, la partida de la dotación de créditos provenientes del Presupuesto de la Comunidad será mucho más relevante y con ello la dependencia económica de la Administración territorial.

Corresponde al Gerente aprobar el borrador de anteproyecto de presupuesto, así como el proyecto de relación de puestos de trabajo de la Agencia. La decisión última, en ambos casos, queda fuera del ámbito de la Agencia.

${ }^{26}$ Aspecto introducido por la enmienda n⿳0 110 del grupo parlamentario del Partido Popular. BOCG. Serie A nº 110-18 de 26 de febrero de 2007.

27 "A greater turnover indicates a lowers level of independence" Cukierman, A. et al "Measuring the independence of central banks and its effects on policy outcomes. World Bank Economic Review, vol. 6 n.3 1992. De hecho, la justificación de la enmienda $n^{\circ} 93$ del grupo parlamentario popular que proponía aumentar a 6 el número de consejeros CNC era precisamente que el aumento del número de consejeros de 4 a 6 supone incrementar la independencia de la Comisión Nacional de Competencia. BOGC. Serie A nํ110-18 de 26 de febrero de 2007. 
Según reconoce el Libro Blanco para la reforma de la defensa de la competencia "las autoridades españolas de competencia disponen de unos medios humanos y materiales claramente menores a los de los países de nuestro entorno" ${ }^{28}$ La escasez de medios humanos no sólo puede resultar un condicionante en la calidad e independencia de la institución sino que en cierta manera configura el tipo de política de competencia que se desarrolla. Así, cuando falta el expertise necesario para abordar los asuntos, lo más fácil es que se recurra a un procedimiento contradictorio entre las partes para hacer aflorar los intereses en juego y el núcleo del asunto sujeto a discusión. Esta preferencia hacia en enfoque contradictorio puede esconder la incapacidad técnica de la institución y provoca que los procedimientos se alarguen más de lo necesario. En tema de fusiones, por ejemplo, la resolución lenta de un expediente puede comprometer la propia transacción.

En definitiva, la dotación de medios humanos altamente especializados y cualificados constituye un elemento crucial tanto en la garantía de independencia como en los procedimientos mismos. De manera reiterada en la redacción de la Ley andaluza 6/2007 aparece la necesidad de que la Agencia cuente con los recursos suficientes para el cumplimiento de sus fines, tanto en medios humanos como materiales.

$\mathrm{El}$ informe OCDE "Regulatory reform in Spain: the role of competition policy in regulatory reform" del año 2000, efectuaba unas valoraciones como mínimo sonrojantes para caso español "el Tribunal no tiene prácticamente ningún personal profesional de apoyo a los miembros. Es improbable que el Tribunal pueda conducir efectivamente cualquiera investigación independiente o suplementaria, sin la asistencia del Servicio o de las partes de un expediente. Una propuesta para aumentar los recursos supone añadir miembros al Tribunal. Pero con nueve miembros, el Tribunal es ya un órgano de toma de decisiones bastante grande. Sin duda, el Tribunal tiene una práctica de hacer rotar las responsabilidades, para usar el tiempo de sus miembros más eficientemente. A pesar de ello, más cuerpo administrativo, antes que más miembros, probablemente tenga un mayor impacto en los resultados y eficiencia. El Servicio se ha reducido en realidad en los últimos años, a pesar de que está manejando el doble de denuncias y pueda revisar ahora un número mayor de las fusiones propuestas, bajo limitaciones de plazo más estrictas. También es probable que el Servicio se vea más forzado, especialmente como consecuencias de las re-

${ }^{28}$ Libro Blanco de 20 de enero de 2005. Disponible en www.dgdc.meh.es (§ 70). 
formas sectoriales que exponen empresas previamente protegidas a las fuerzas competitivas." ${ }^{29}$

Esta apreciación coincide plenamente con la valoración que efectúa la publicación especializada más autorizada en materia de competencia en España. Efectivamente, el Anuario de la Competencia 2003 describe que "el descenso progresivo de las denuncias en los últimos años no se ha visto acompañado de un impulso de la actividad de persecución de oficio de las autoridades. Si lo observamos respecto a los últimos cuatro años (2000 a 2003) puede verse cómo los expedientes de prácticas restrictivas derivados de denuncia que valora el Servicio se han reducido (99, 95, 70 y 53) y los expedientes de oficio también se han reducido $(3,1,2$ y 0$)$. Seguramente el volumen de las denuncias sufre un proceso lógico de adaptación. Buena parte de las denuncias que se presentaban eran objeto de archivo inmediato, de forma que es posible pensar que progresivamente el volumen se ajusta a una aplicación real de las normas de competencia. No obstante, resulta cuestionable la inexistencia de una aplicación de oficio de las normas de competencia. Únicamente se actúa entonces en función de la existencia de denuncias, lo que no parece de recibo". ${ }^{30}$

El desarrollo y garantía de la independencia de los organismos de defensa de la competencia pasa pues por la consiguiente dotación humana de la nueva institución, lo que al final puede resultar igual o más crucial que el diseño institucional mismo.

\subsection{Promoción de la competencia (competition advocacy)}

La función de promoción de la competencia (competition advocacy en terminología anglosajona), constituye un elemento clave en el esfuerzo de transmitir a la sociedad los beneficios de la libre y leal competencia así como el desvalor de las conductas que la alteren o falseen. Se trata de implicar también a la sociedad en la lucha por los mercados y por el respeto a la posición del consumidor.

En lo competencial, hemos visto como la distribución de competencias permite distinguir entre la competencia andaluza exclusiva en materia de pro-

${ }^{29}$ OECD Regulatory reform in Spain. The role of Competition Policy in Regulatory Reform. París 2000. Policy conclusions, p. 33.

${ }^{30}$ CASES PALLARÉS, Ll. "La competencia en España 2003" Anuario de la competencia 2003. ICO-Marcial Pons, Madrid, 2004, p. 15. 
moción de la competencia y la competencia ejecutiva en materia de defensa de la competencia. La posición de las Comunidades es, por ello, más relevante frente al Estado en lo que a competition advocay se refiere.

En estrecha relación con lo anterior, no hay que desdeñar tampoco la potencialidad del principio de transparencia como garantía de independencia. Así, el informe OCDE sobre el diseño de instituciones independientes establece que la independencia puede basarse en un doble diálogo: de una parte entre la institución y el Parlamento (informes anuales), y de otra entre la institución y la ciudadanía (mecanismos de publicidad de los expedientes e informes $)^{31}$

El modelo estatal recupera la publicidad de los informes sobre la estructura competitiva de mercados o sectores productivos, que gozaron de notable impacto en años precedentes. Junto con esto, paradójicamente, no se crea una Dirección de estudios que sea responsable de la elaboración de los mismos.

Al contrario, la Agencia Andaluza sí crea de manera digna de encomio una Dirección de Estudios, Análisis de Mercados y Promoción de la Competencia. En cambio, no se aseguran expresamente para esta Dirección los medios que sí se asignan al Departamento de Investigación, ${ }^{32}$ ni queda explicitada la publicidad de los informes, más allá de entenderlos incluidos en el concepto de "demás actos y actuaciones en materia de defensa de la competencia" del artículo 6.1.

Las deficiencias en uno y otro modelo son palpables pero asimétricas: gran refuerzo de la publicidad de los informes sin establecer un Departamento para ello (Estado) o creación específica de un Departamento sin dotarlo de medios y si asegurar la publicidad de los informes (Andalucía).

31 OECD Working Party on regulatory management and reform. Designing independent and accountable regulatory authorities for high quality regulation. 2005. La legitimidad técnica de las Administraciones Independientes no significa su total desvinculación con la sociedad democrática que deben regir.

${ }^{32}$ En la regulación del Departamento de Investigación se establece que: "Para el ejercicio de sus competencias, la Dirección del Departamento de Investigación de Defensa de la Competencia de Andalucía, contará con el suficiente apoyo jurídico y económico de carácter estrictamente profesional e independiente" (art. 19.3.). 


\section{Cuadro comparativo}

\begin{tabular}{|l|c|c|}
\hline naturaleza & Comisión Nacional & Agencia andaluza \\
\hline independencia & $\begin{array}{c}\text { Administración } \\
\text { independiente }\end{array}$ & $\begin{array}{c}\text { Agencia } \\
\text { administrativa }\end{array}$ \\
\hline integración orgánica & Sí & formal \\
\hline miembros & Sí & Sí \\
\hline hearing parlamentario & 7 & 3 \\
\hline requisitos de profesionalidad & Sí & No \\
\hline mandato & Sí & Sí \\
\hline gerente & 6 & $5+5$ \\
\hline dirección de estudios & No & Sí \\
\hline rendición de cuentas & No & Sí \\
\hline
\end{tabular}

\section{CONCLUSIONES}

La descentralización de la política de defensa de la competencia en España a partir de la STC 208/99 ha dado lugar al nacimiento de varias autoridades autonómicas de defensa de la competencia. En este sentido, es destacable como no se ha utilizado la técnica de la participación autonómica en las instituciones nacionales para dar cauce a la posición de las Comunidades. En definitiva, el modelo descentralizado desemboca en una doble red de autoridades estatales y autonómicas de defensa de la competencia que aplican la Ley de manera coordinada.

En razón de este proceso, el Parlamento de Andalucía ha aprobado la Ley 6/2007, de 26 de junio, de Promoción y Defensa de la Competencia de Andalucía. La norma prevé la creación de una Agencia con una estructura compleja que divide entre Dirección-Gerencia; Consejo, Departamento de Investigación; Departamento de Estudios y Secretaría General.

La regulación de las figuras del Presidente y del Gerente puede dar lugar a una cierta tensión en el liderazgo de la Agencia. Si bien es verdad que la representación legal y la dirección corresponden al Gerente, la competencia por razón de la materia y las facultades resolutorias se sitúan en el Presidente. 
En el caso andaluz, el organismo de defensa de la competencia se configura como una Agencia cuya naturaleza es la de un organismo autónomo de carácter administrativo que está adscrito a la Consejería competente en materia de economía. No se trata como en la Comisión Nacional de Competencia de una verdadera autoridad independiente y no hay ningún mecanismo de garantía de la independencia más allá de los constantes llamamientos que hace la Ley. El nombramiento de los miembros de la Agencia no se somete a ningún trámite parlamentario y su única garantía es un mandato de duración superior al de una legislatura.

El Consejo Económico y Social de Andalucía ya advertía de este aspecto en el Dictamen del texto del anteproyecto de manera que "será fundamental que la norma garantice la independencia y transparencia de este Órgano, así como la capacidad profesional de sus miembros, por lo que habrían de incluirse las especificaciones necesarias a este respecto, que aseguren la viabilidad y eficiencia de la Agencia de promoción y defensa de la competencia en Andalucía". 33

La legislación estatal acompaña la unidad orgánica y la independencia con el establecimiento de mecanismos de rendición de cuentas ante el Parlamento que, a pesar de existir, tienen una intensidad algo menor en la Comunidad andaluza.

La autonomía económica de la Agencia andaluza, aunque formalmente es parecida a la de la Comisión Nacional, en el fondo queda reducida al no poder contar con los ingresos propios que genera la tasa por análisis y estudio de las operaciones de concentración.

En promoción de la competencia, se produce el absurdo de que la institución que cuenta con una estructura explícita para realizar la función, no asegura la publicidad de los resultados. Al contrario, el Estado enuncia la publicidad de los informes de mercados y sectores pero no dispone específicamente de una Dirección de Estudios.

Todos estos elementos, y en especial la diferente formulación y garantía de la independencia de la institución nos llevan a preguntarnos sobre el impacto del modelo estatal sobre el andaluz. Formalmente, la Ley andaluza se

\footnotetext{
${ }^{33}$ Dictamen CES Andalucía no 7/2006 sobre el Anteproyecto de Ley de Promoción y Defensa de la Competencia de Andalucía, 8 mayo 2006.
} 
aprueba con anterioridad a la estatal (aunque tan breve que ambas normas aparecen en los Boletines oficiales respectivos del mismo día 4 de julio de 2007). Además, la norma estatal no prevé que el modelo CNC sea básico y por tanto de adopción obligatoria por parte de las Comunidades. Lo que sucede, sin embargo, es que algunas de las funciones que prevé la Ley 15/2007 no son fácilmente realizables sin una garantía real de la independencia del organismo.

Ello sucede, por ejemplo, respecto del informe de las autoridades autonómicas sobre ayudas públicas o sobre la fenomenal potestad de los órganos autonómicos para la impugnación de disposiciones administrativas ante la jurisdicción cuando consideren que vulneran la libre competencia ${ }^{34}$.

En todos estos casos, se pondría de manifiesto la vis expansiva del modelo estatal de Comisión independiente más allá de la aplicabilidad misma del artículo 149.1.18 de la Constitución.

\section{BIBLIOGRAFÍA}

ARZOZ SANTISTEBAN, X. "El impacto de los puntos de conexión de la Ley 1/2002 en la aplicación descentralizada del derecho de la competencia: análisis de las resoluciones adoptadas en 2003 por el TDC" Anuario de la Competencia 2003. ICO-Marcial Pons, Madrid, 2004.

BALLBÉ MALLOL, M. y PADRÓS REIG, C. "Spanish independent authority and its role in a new competitive environment" en AMATO, G. and LAUDATI, L.L. (eds.) The anticompetitive impact of regulation. London, 2001.

BENEYTO PÉREZ, J.M. y MAILLO GONZÁLEZ-ORÚS, J. El nuevo derecho comunitario y español de la competencia: descentralización, análisis económico y cooperación internacional. Ed. Bosch, Barcelona 2002.

BENEYTO PÉREZ, J.M. y MAILLO GONZÁLEZ-ORÚS, J. Tratado de derecho de la competencia: Unión Europea y España Ed. Bosch, Barcelona 2005.

CASES PALLARÉS, Ll. Derecho administrativo de la defensa de la competencia. Marcial Pons. Madrid, 1995.

\footnotetext{
${ }^{34}$ Los Estatutos prevén que sea competente para impugnar el Consejo previo acuerdo de la Secretaría General que es un órgano nombrado por el Gerente, a su vez nombrado por el Gobierno (art. 8.3.c. Decreto 289/2007 de 11 de diciembre). Lo mismo puede decirse respecto a la promoción de la competencia en cuanto los sectores a analizar sean públicos. Ello no es una cuestión menor si tenemos en cuenta que una de las fuentes de vulneración más frecuente de la competencia es precisamente la actuación del poder público o de sus sociedades instrumentales.
} 
CASES PALLARÉS, Ll. Anuario de la competencia 2003. ICO-Marcial Pons. Madrid 2004.

NADAL, M. y ROCA, J. La descentralización de la política de defensa de la competencia en España: situación y perspectivas. Instituto de la Empresa Familiar. Barcelona, 2003.

OECD Regulatory Reform in Spain. The Role of Competition Policy in Regulatory Reform. París 2000.

OECD Global Forum on Competition. Optimal Design of a Competition Agency, 2003.

PADRÓS REIG, C. "Un nuevo marco institucional de la defensa de la competencia en España" Universidad San Pablo CEU. Instituto Universitario de Estudios Europeos-Centro de política de la competencia. Documento de trabajo, Serie política de la competencia 19/2006.

PADRÓS REIG, C. "El esquema institucional" en MAILLO GONZÁLEZORÚS, J. (coord.) Comentarios a la Ley 15/2007 de defensa de la competencia. Editorial Bosch, Barcelona, 2008.

PADRÓS REIG, C. "The new institutional framework of Spanish competition Policy” European Competition Law Review vol. 28, nํ 12, December 2007.

PADRÓS REIG, C. "Los organismos autonómicos de defensa de la competencia. Situación y perspectivas" Anuario de la competencia. En prensa. 2008.

PEÑAFIEL GARCÍA, G. "La aplicación práctica de la Ley 1/2002" Anuario de la Competencia 2004. ICO-Marcial Pons, Madrid, 2005.

SOLANA GONZÁLEZ, G. "Pasado, presente y futuro del modelo español de defensa de la competencia" Anuario de la Competencia 2003. ICO-Marcial Pons, Madrid, 2004.

SOLANA GONZÁLEZ, G. "Unas bases sólidas para acometer la reforma" Anuario de la Competencia 2004. ICO-Marcial Pons, Madrid, 2005. 